\title{
Small-scale cloud processes and climate
}

\author{
Marcia B. Baker \& Thomas Peter
}

\section{Clouds constitute the largest single source of uncertainty in climate prediction. A better understanding of small-scale cloud processes could shed light on the role of clouds in the climate system.}

Clouds control Earth's weather and regulate its climate ${ }^{1,2}$. They cool Earth's atmosphere by reflecting incoming visible-wavelength solar radiation and warm its surface by trapping outgoing infrared radiation. Clouds produce the rain and snow that dominate Earth's weather and shape Earth's landscapes and vegetation zones.

The large-scale effects of clouds are difficult to characterize accurately because they result from processes that occur on very small scales ${ }^{3}$. Small particles, ranging in size from nanometres to hundreds of micrometres, are strongly affected by updraughts and downdraughts and turbulent mixing on scales of metres to kilometres. Figure 1 shows schematically how largescale cloud properties depend on small-scale processes. Submicrometre aerosol particles produced by natural processes, such as dust storms, and by anthropogenic processes, such as burning of wood and fuel, constitute the nuclei on which water droplets and ice crystals form in a cloud. Traditionally thought to consist mainly of sulphates ${ }^{4}$, aerosols are now known to have a much more varied composition. Cloud particles in the form of water droplets or ice crystals then grow by taking up water vapour. The radiative property of clouds depends on the size and the number of cloud particles. If the cloud particles reach tens of micrometres, they fall rapidly, colliding with one another to form rain.

The rates at which cloud particles form, grow and fall out of clouds depend on the concentrations, sizes and chemical compositions of the aerosol particles. They also depend on the humidity, temperature and vertical velocity of the air, and on the fluctuations in these parameters (over a distance of 100 metres to several kilometres). Anthropogenic modification of the concentrations and/or chemical compositions of aerosol particles might therefore influence cloud development, weather and climate. An example of this is shown in Fig. 2; in this satellite photograph of clouds over the Atlantic Ocean, the thin white lines crossing the image are bright clouds consisting of small drops that form on the particles emitted by ships, a particularly vivid demonstration of human activity altering the reflectivity of Earth.

\section{Recent developments}

Over the past few decades, the ability to observe small-scale cloud phenomena has improved markedly. Sophisticated laboratory equipment allows the observation of individual micrometre-sized particles suspended in the air. In addition, satellite-borne instruments can now detect, and to some extent identify, cloud and aerosol particles. With these developments, there have been incredible achievements - but new challenges have also been presented.

Remarkable progress has been made in understanding how aerosol particles modify droplet freezing in clouds. Freezing has tremendous climatic effects because it is often the first step in rain formation. It modifies the rate of cloud ascent, and freezing in the upper troposphere creates cirrus clouds, which are thin clouds (composed of ice crystals) that are effective at trapping outgoing radiation.

It might seem surprising that there is more to learn about freezing. Although bulk freezing near $0{ }^{\circ} \mathrm{C}$ is well understood, water in tiny droplets can persist as a supercooled liquid at much lower temperatures.

It was traditionally thought that formation of ice in clouds required solid aerosol particles known as ice nuclei. But it is now known that at temperatures below $-35^{\circ} \mathrm{C}$, most ice forms spontaneously through 'homogeneous' freezing in aqueous droplets that contain no foreign particles. The rate of this process depends on, and can be predicted from, air temperature, humidity and small-scale vertical air motions, but is rather insensitive to the chemical composition of the pre-existing aqueous aerosol droplets ${ }^{5}$.

By contrast, ice formation at temperatures between $0{ }^{\circ} \mathrm{C}$ and $-35^{\circ} \mathrm{C}$ can be initiated only through heterogeneous nucleation. Ice nuclei such as mineral-dust particles were originally thought to be inert. But laboratory studies have shown that physicochemical transformation of other aerosol particles, such as those containing organic material, can modify how efficiently ice nuclei function as freezing substrates. This, in turn, makes it difficult to identify the origin of the ice nuclei involved in cloud formation and to predict the climatic role of clouds.

\section{Current challenges}

Because cloud processes are sensitive to the concentration and type of aerosol particle, a major and controversial focus of recent

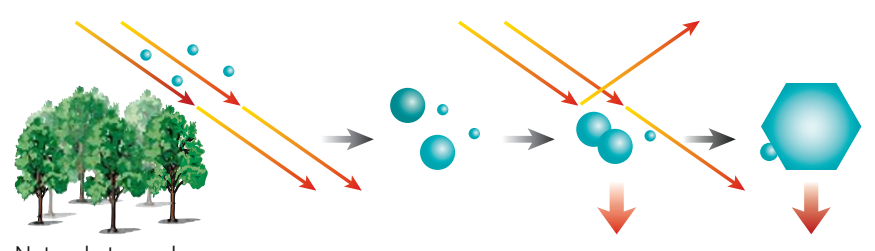

Natural atmosphere

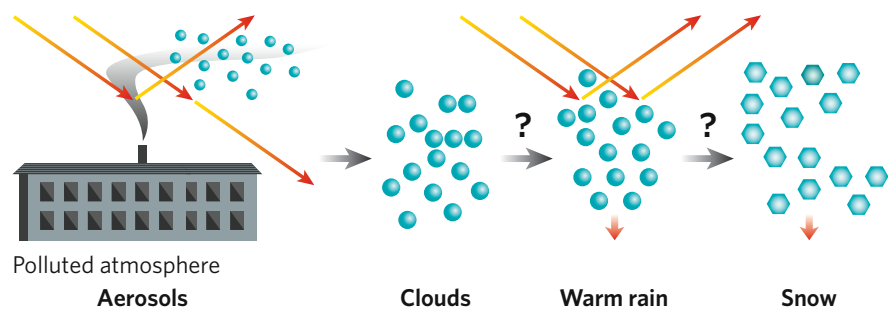

Figure 1 | Interactions of aerosol particles with clouds and the consequences for cloud development. In the natural (non-polluted) atmosphere, the concentrations of aerosol particles are generally low, and the clouds that form on these particles have relatively low droplet and/or ice-crystal concentrations. The increased concentrations of aerosol particles present in polluted atmospheres might lead to the formation of clouds with high concentrations of droplets or ice crystals. Such clouds are expected to reflect more radiation than clouds with fewer droplets ${ }^{6}$, as is corroborated by an increasing body of observational evidence. High cloud-particle concentrations can lead to smaller droplet or crystal sizes and therefore to reduced particle fall speeds (shown by the vertical red arrows; the larger the arrow, the faster the fall). This effect, acting alone, would increase the rate of fallout of precipitation (snow or rain) in non-polluted environments. By contrast, in polluted environments, it would tend to increase the time for which the cloud particles remain suspended ${ }^{7}$. However, observational evidence for aerosol effects on large-scale changes in cloud lifetimes and precipitation patterns is still lacking (as shown by the question marks). 


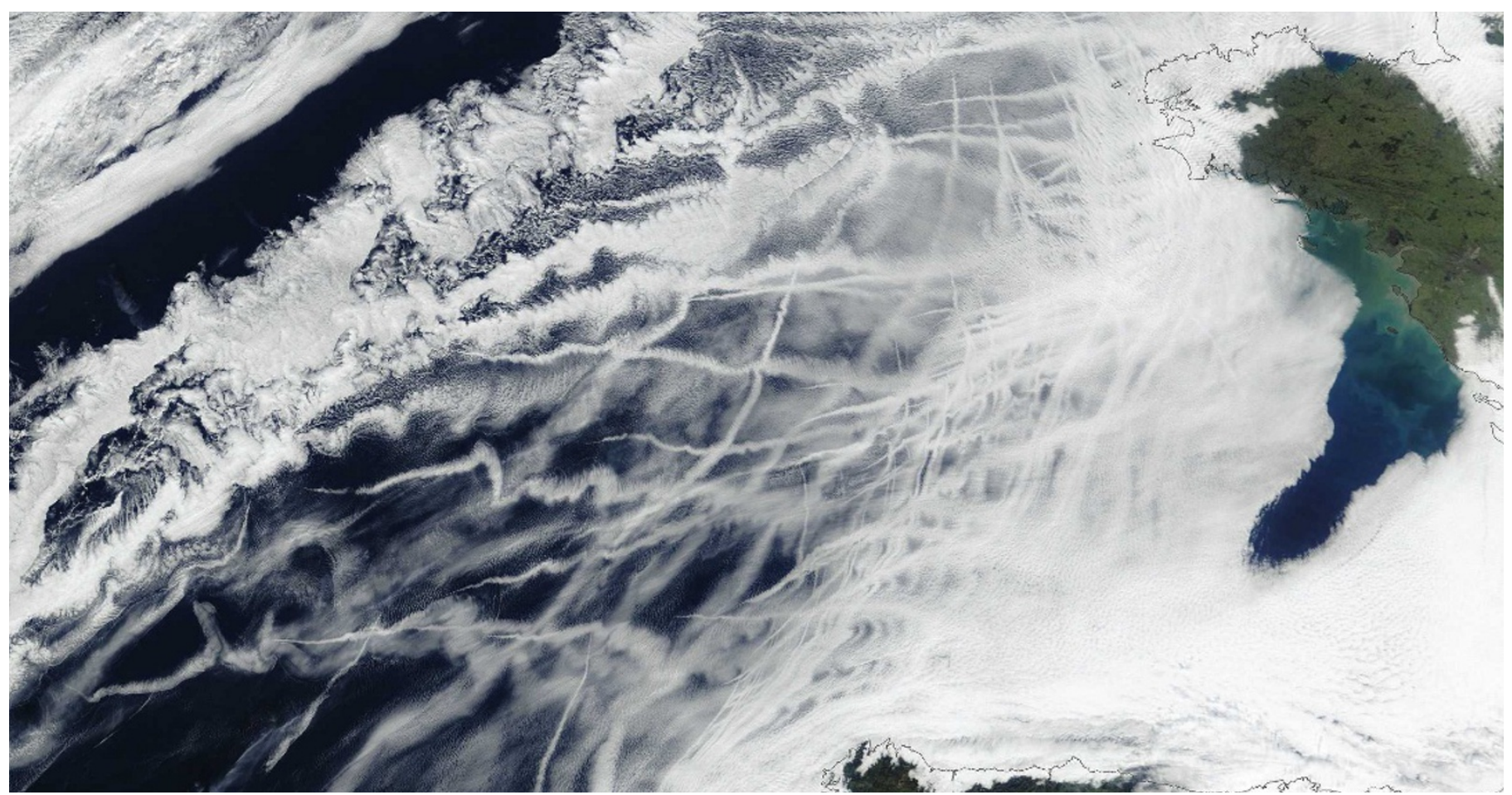

Figure 2 Satellite photograph of low clouds over the Atlantic Ocean. The thin white lines are locally enhanced clouds formed in tracks marking the effluent from smokestacks on passing ships. (Image courtesy of J. Descloitres, MODIS Rapid Response Team, NASA/GSFC, Greenbelt, Maryland. )

cloud research has been the attempt to quantify the extent to which anthropogenic aerosol particles are modifying cloud properties on a global scale. As indicated in Figs 1 and 2, high concentrations of aerosols can increase the brightness of clouds and their ability to reflect solar radiation, a process that is moderately well understood ${ }^{6}$ but not well quantified globally. Moreover, it has been suggested that an increased aerosol concentration alters large-scale patterns in cloud lifetimes and precipitation ${ }^{7}$, but these effects of aerosol particles are highly uncertain at present ${ }^{1}$.

New observational programmes ${ }^{1}$ and numerical model approaches ${ }^{8}$ will be required to pin down the effect of anthropogenic aerosol particles on large-scale cloud properties. Such programmes should include dedicated laboratory investigations of cloud-particle formation and field studies that measure small-scale parameters and follow cloud development over extended periods of time. These process-oriented approaches need to be tightly linked with satellite and surface-based networks that monitor the important cloud variables with sufficient precision and stability to quantify accurately any changes over the coming years to decades.

These improvements will provide exciting intellectual and practical benefits as scientists become increasingly able to predict the development of individual clouds and cloud systems, which has been the goal of much atmospheric research over the past 50 years. Future research into small-scale cloud processes will yield new insights into the largescale phenomena that characterize Earth's climate.
Marcia B. Baker is in the Department of Earth and Space Sciences, University of Washington, Seattle, Washington 98195, USA. Thomas Peter is at the Institute for Atmospheric and Climate Science, ETH Zürich, 8092 Zürich, Switzerland.

1. Solomon, S. et al. (eds) Climate Change 2007: The Physical Science Basis. Contribution of Working Group I to the Fourth Assessment Report of the Intergovernmental Panel on Climate Change (Cambridge Univ. Press, Cambridge, UK, 2007).

2. Collins, W., Colman, R., Haywood, J., Manning, M. R. \& Mote, P. The physical science behind climate change. Sci. Am. 297, 64-73 (2007).

3. Baker, M. B. Cloud microphysics and climate. Science 276, 1072-1078 (1997)

4. Charlson, R. J. \& Wigley,T. M. L. Sulfate aerosol and climatic change. Sci. Am. 270, 48-57 (2004).

5. Koop, T., Luo, B. P., Tsias, A. \& Peter, T. Water activity as the determinant for homogeneous ice nucleation in aqueous solutions. Nature 406, 611-614 (2000).

6. Twomey, S. Influence of pollution on the short-wave albedo of clouds. J. Atmos. Sci. 34 1149-1152 (1977)

7. Albrecht, B. A. Aerosols, cloud microphysics and fractional cloudiness. Science $\mathbf{2 4 5}$ 1227-1230 (1989)

8. Lohmann, U., Quaas, J., Kinne, S. \& Feichter, J. Different approaches for constraining global climate models of the anthropogenic indirect aerosol effect. Bull. Am. Met. Soc. 88 243-249 (2007)

Acknowledgements M.B.B. is grateful to R. Wood and G. Raga for helpful comments. T.P. thanks the European Commission and the Swiss National Foundation for financial support.

Author Information Reprints and permissions information is available at npg.nature.com/reprints. Correspondence should be addressed to M.B.B. (marcia@ess.washington.edu). 